\title{
La vertebroplastica e la cifoplastica nel paziente uremico cronico con crollo vertebrale
}

\section{R. Partescano}

S.C. Ortopedia e Traumatologia, Ospedale S. Maria Annunziata Azienda Sanitaria di Firenze

La perdita di massa ossea è una complicanza tipica e costante del paziente affetto da insufficienza renale cronica (IRC) (1). Tale depauperamento inizia precocemente nella storia dell'IRC, tanto che il rischio fratturativo è già aumentato nello stadio 2 DOQI (Tab. I) per poi peggiorare progressivamente negli stadi successivi fino a divenire massimo nei pazienti in trattamento dialitico cronico, in cui risulta 17 volte maggiore rispetto a quello della popolazione generale $(1,2)$. Anche i pazienti sottoposti a terapia steroidea a lungo termine, come i portatori di trapianto renale, sono ad alto rischio di fratture.

La progressiva perdita di matrice minerale ossea e le alterazioni strutturali dell'osso rendono i corpi vertebrali dei pazienti uremici cronici molto vulnerabili. Traumi anche lievi possono provocare fratture vertebrali da compressione che, come in ogni paziente gravemente osteoporotico, costituiscono una pesante causa di disabilità e possono causare un aumento della morbilità e della mortalità (Fig. 1) (3).

Anche la qualità della vita è fortemente intaccata nei pazienti che subiscono un crollo vertebrale. Oltre al dolore, che può essere altamente invalidante e permanere per mesi fino alla sua stabile cronicizzazione (4), molti altri fattori (Fig. 1) portano il paziente a una ridotta autonomia psico-fisica, innescando meccanismi di dipendenza, depressione, limitazione del proprio ruolo nella società e grave riduzione dell'autostima (5).

Fino ad alcuni anni fa il trattamento di queste fratture prevedeva spesso la necessità di lunghi periodi di riposo a letto, con frequentissima insorgenza di sindrome da immobilizzazione, oppure l'applicazione di busti ortopedici che, limitando notevolmente le comuni attività quo- tidiane, riducevano il tono muscolare paravertebrale esponendo i pazienti al rischio di ulteriori conseguenze indesiderate. Inoltre, l'uso di analgesici era pressoché obbligato per lunghi periodi e associato ad alto rischio di effetti collaterali da farmaci anti-infiammatori non

TABELLA I - STADI DELL'IRC (Am J Kidney Dis 202; 39 - S1)

$\begin{array}{ll}\text { - } \text { STADIO } 1 & \mathrm{VFG} \geq 90 \mathrm{ml} / \mathrm{min} \\ \text { - STADIO 2 } & \text { VFG } 60-89 \mathrm{ml} / \mathrm{min} \\ \text { - STADIO } 3 & \text { VFG } 30-59 \mathrm{ml} / \mathrm{min} \\ \text { - STADIO 4 } & \text { VFG } 15-29 \mathrm{ml} / \mathrm{min} \\ \text { - STADIO 5 } & \text { VFG }<15 \mathrm{ml} / \mathrm{min}\end{array}$

Il rischio fratturativo è aumentato anche in presenza di una modesta compromissione renale ovvero stadio 2-3 ed aumenta progressivamente all'aumentare di severità della IRC, risultando particolarmente presente nei pazienti in stadio 4 e 5 .

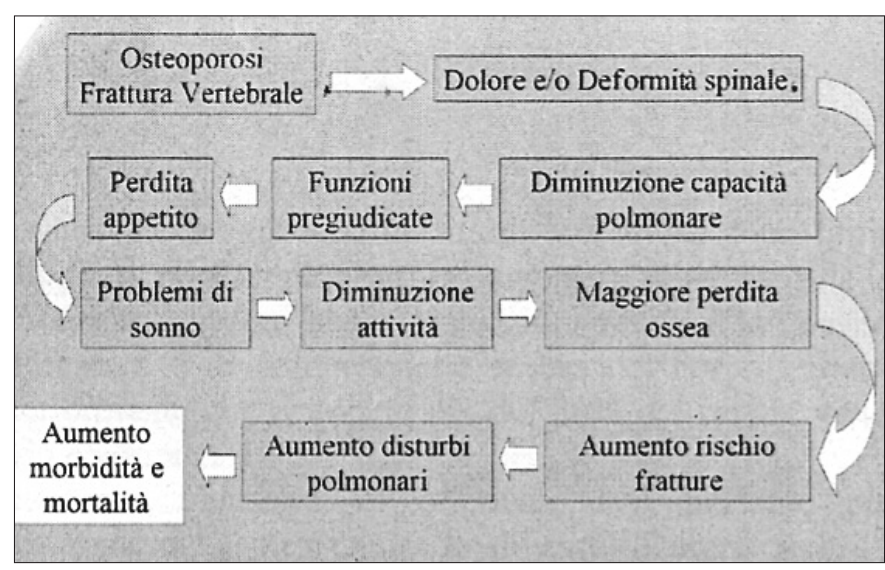

Fig. 1 - Possibili conseguenze delle fratture vertebrali (3). 


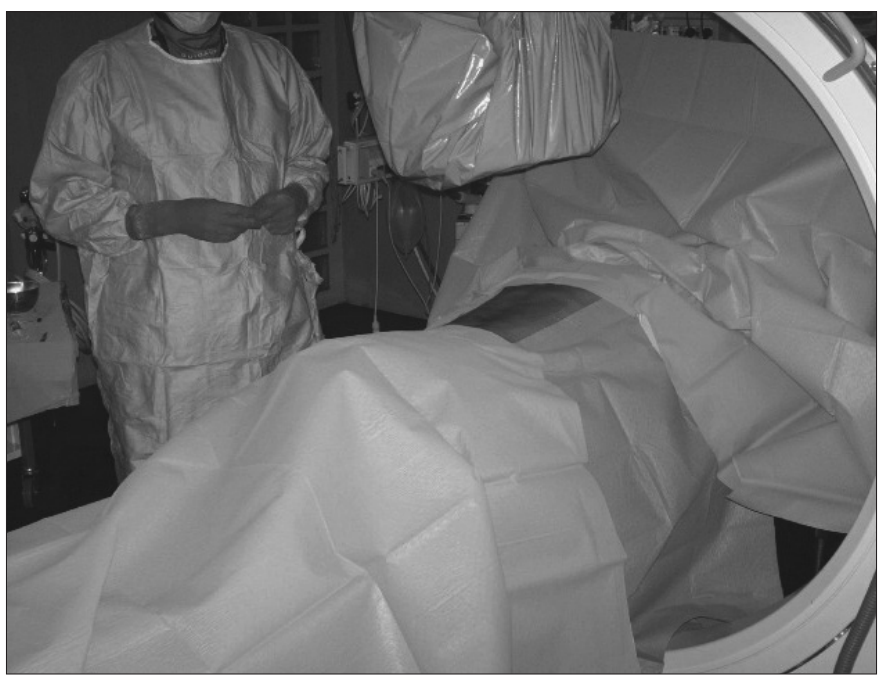

Fig. 2 - Campo operatorio.

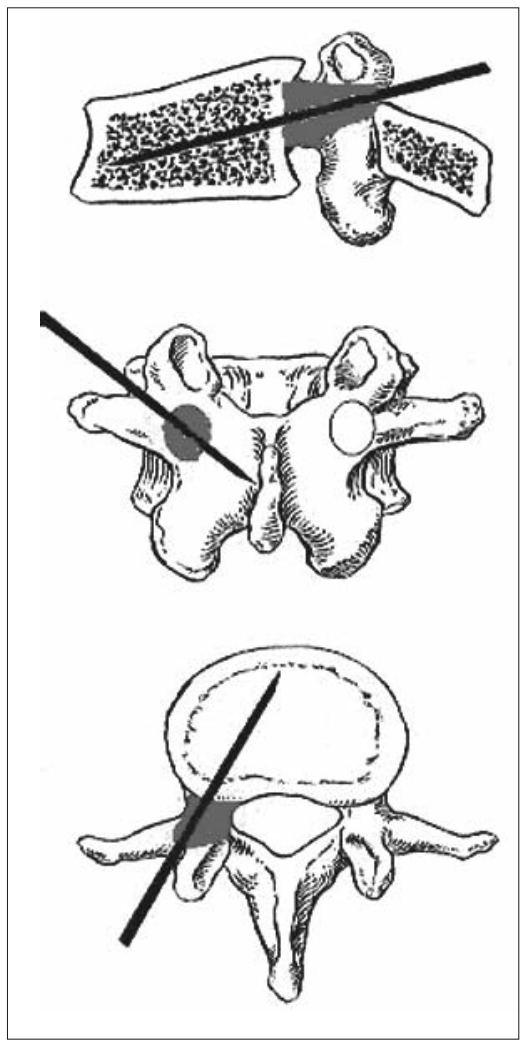

Fig. 4 - Accesso nel corpo vertebrale attraverso la via transpeduncolare. In grigio il peduncolo vertebrale.

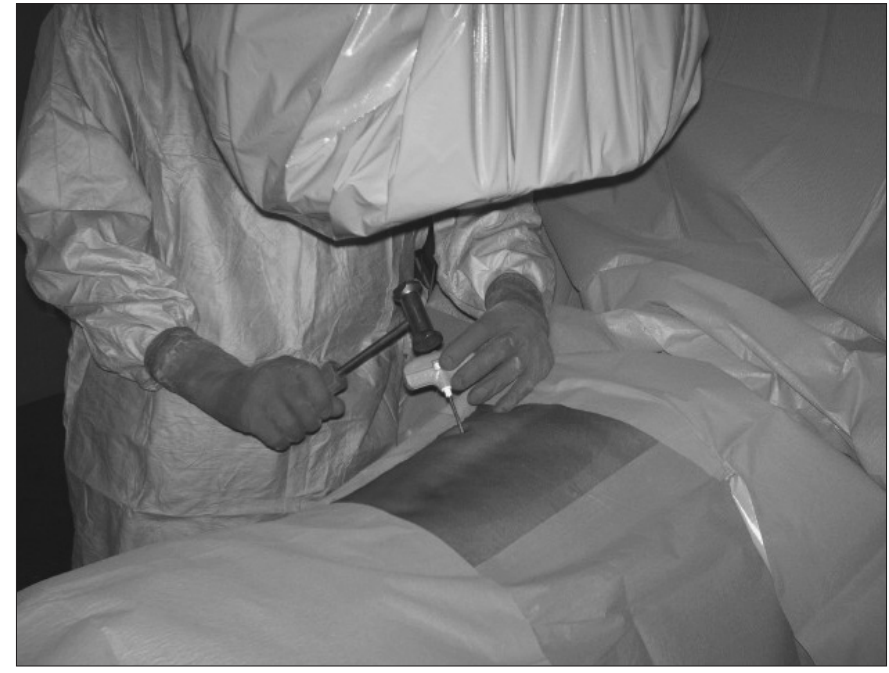

Fig. 3 - Introduzione trocar sotto controllo amplioscopico.

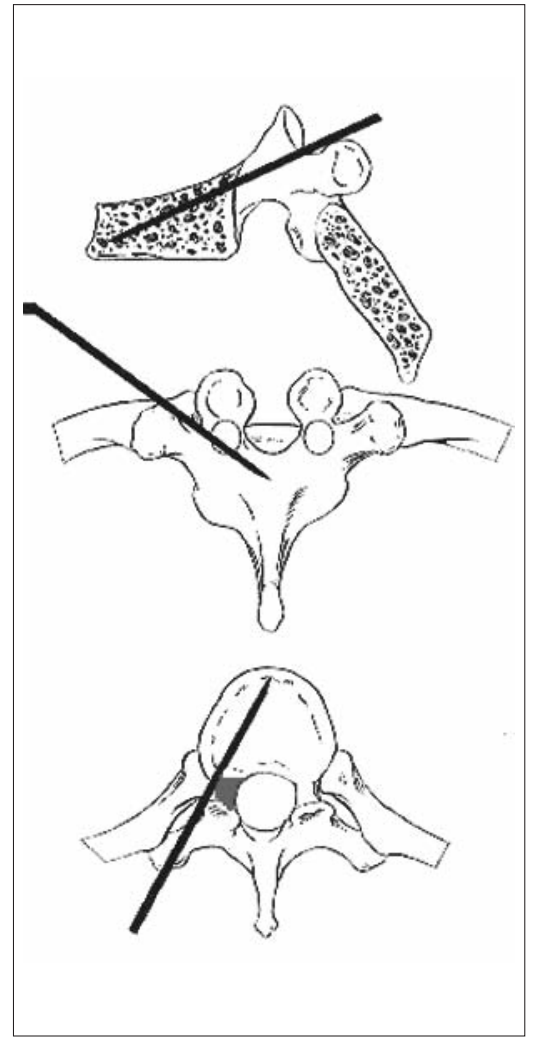

Fig. 5 - Accesso extrapeduncolare: il suo utilizzo per le vertebre dorsali più alte permette di inclinare maggiormente il trocar nonostante le ridotte dimensioni del peduncolo. steroidei (FANS), primo fra tutti quello di gastroenteropatie emorragiche.

Da qualche anno il trattamento conservativo di queste fratture è stato affiancato dall'uso di due metodiche miniinvasive, la vertebroplastica (6) e la cifoplastica (7). La prima metodica a essere utilizzata nella pratica clinica è stata la vertebroplastica percutanea (6). Questa consiste nell'introduzione di cemento osseo radiopaco all'interno del corpo vertebrale fratturato attraverso un ago metallico posizionato per via transpeduncolare sotto controllo amplioscopico. Questa tecnica è stata poi migliorata con l'avvento del sistema "Confidence" che, mediante una pompa pneumatica, permette l'inserimento di un cemento ad alta viscosità riducendone co- 


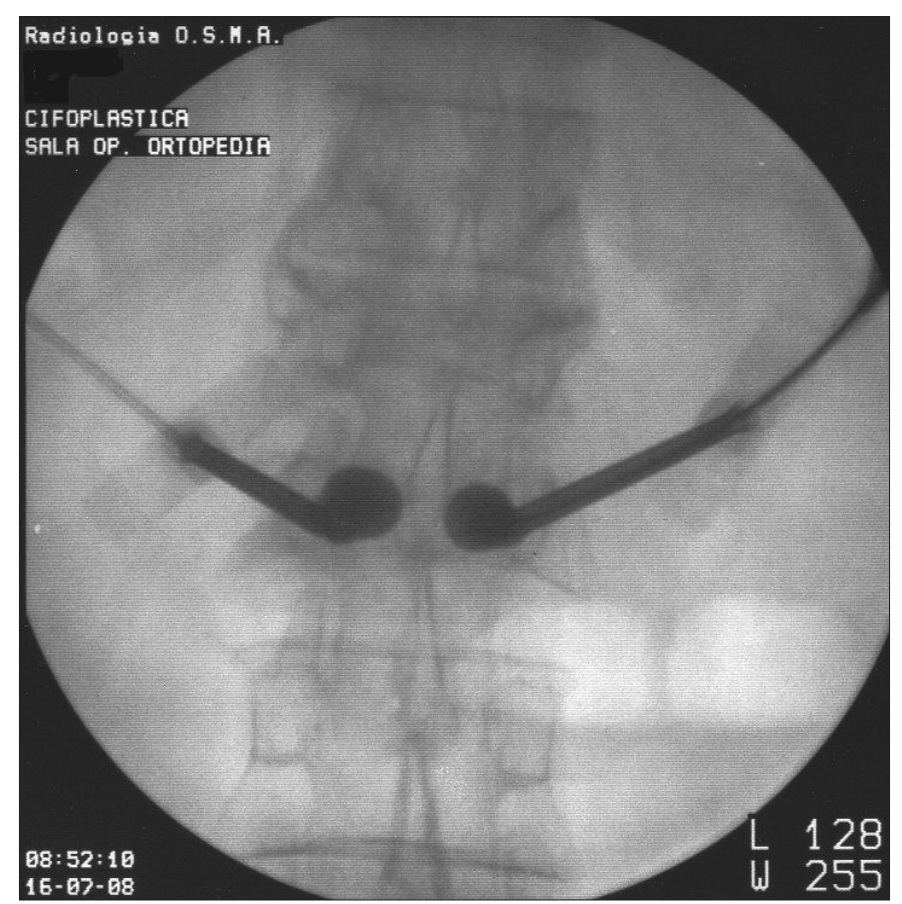

Fig. 6 - Riempimento dei palloncini all'interno del corpo vertebrale per creare la cavità che conterrà il PMMA.

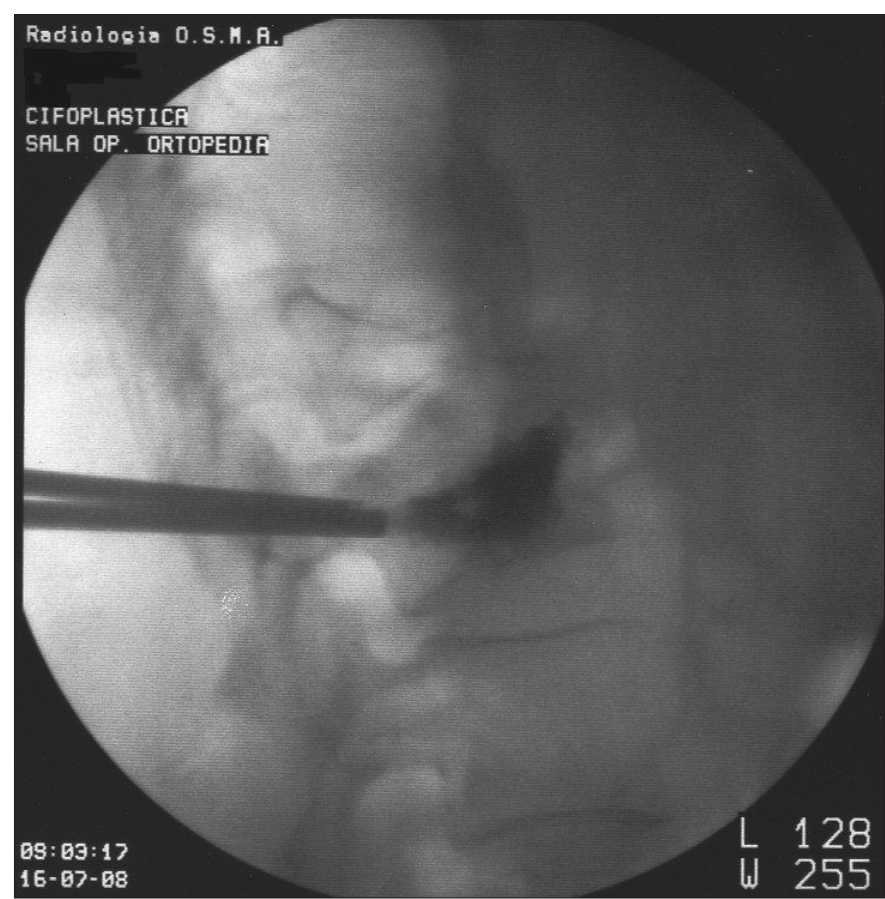

Fig. 7 - Controllo in scopia durante l'introduzione del PMMA all'interno del corpo vertebrale.

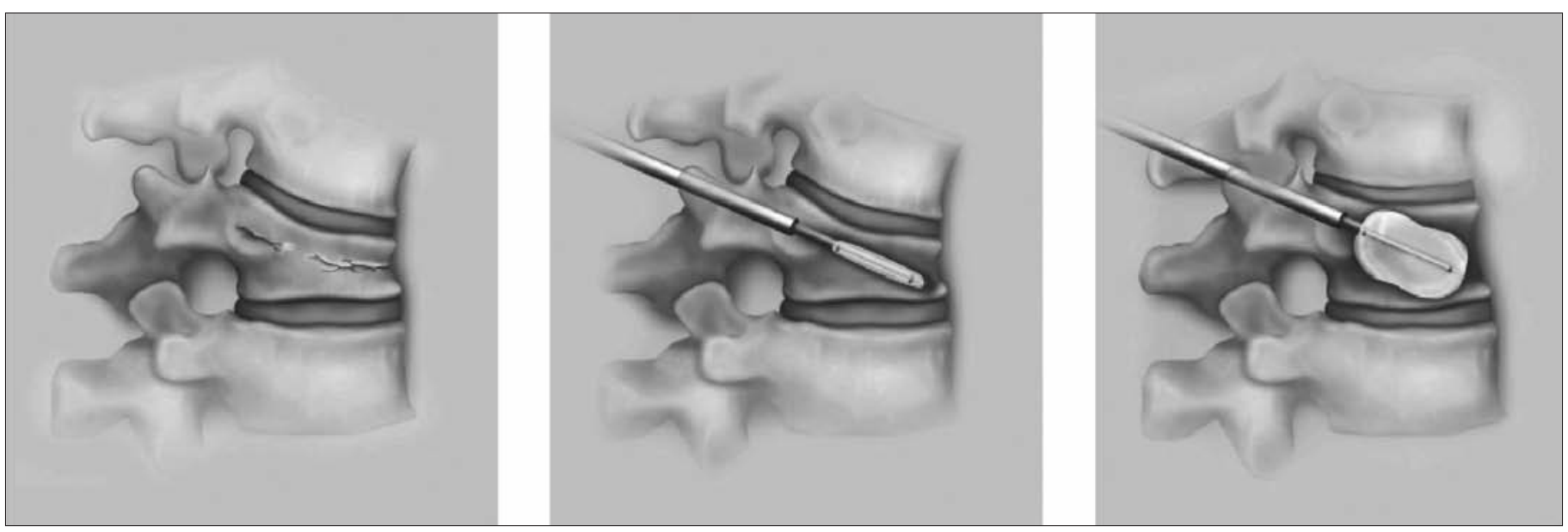

Fig. 8 - Riduzione della frattura attraverso il gonfiaggio del palloncino all'interno del corpo vertebrale.

sì il rischio di fuoriuscita dal corpo vertebrale. La cifoplastica percutanea (8), entrata in uso successivamente, prevede invece l'introduzione del cemento osseo radiopaco all'interno di una cavità creata appositamente (nel corso della stessa seduta) allinterno del corpo vertebrale mediante un palloncino simile a quello impiegato per le angioplastiche percutanee. Entrambe le metodiche hanno lo scopo di "irrobustire e/o incollare" la vertebra fratturata introducendovi un collante oppure cemento acrilico polimetilmetacrilato
(PMMA) simile a quello utilizzato in Ortopedia per fissare le protesi d'anca all'osso $(6,9,10)$.

La principale indicazione alla plastica vertebrale è rappresentata dalle fratture vertebrali lombari o dorsali da schiacciamento resistenti alla terapia conservativa, associate a dolore persistente e con caratteristiche radiologiche di fratture recenti (evidenza di edema e rimaneggiamento osseo alle immagini STIR della RMN, cioè immagini in cui si sottrae il tessuto adiposo e si evidenzia l'edema post-fratturativo). Tali fratture sono 
usualmente secondarie a osteoporosi, traumi o lesioni osteolitiche neoplastiche, in particolare da mieloma multiplo o metastasi ossee.

Le controindicazioni assolute a tali metodiche sono limitate alla gravidanza, a coagulopatie non correggibili, ad allergie verso i componenti utilizzati, alle infezioni sistemiche o locali.

Costituiscono invece controindicazioni relative la coesistenza di dolore radicolare per sindrome compressiva o di compressione severa del corpo vertebrale, la presenza di frammenti dislocati posteriormente che occupino più del $30 \%$ del canale vertebrale o di tumori estesi all'interno dello spazio epidurale.

Lintervento chirurgico è effettuato con paziente posizionato prono su tavolo operatorio radiotrasparente, sotto controllo di amplificatore di brillanza e nella maggior parte dei casi in anestesia locale (Fig. 2). Attraverso una o due (accesso mono- o bipeduncolare) piccole incisioni cutanee paravertebrali di circa $5-6 \mathrm{~mm}$ viene fatto procedere un trocar (Fig. 3) fino a farlo arrivare all'interno del corpo vertebrale (11). Il passaggio del trocar può avvenire per via trans- (Fig. 4) o extrapeduncolare (Fig. 6) a seconda del livello vertebrale. Generalmente l'accesso trans- peduncolare è utilizzato per le vertebre lombari e per le ultime toraciche (T10-T12). Per le altre vertebre toraciche è preferibile l'accesso extrapeduncolare in quanto i peduncoli hanno un diametro minore e può essere più difficoltoso passarvi all'interno. Posizionato il trocar all'interno del corpo vertebrale, nella vertebroplastica si procede direttamente all'immissione del cemento. Nella cifoplastica, invece, a questo punto si inserisce un palloncino che viene gonfiato all'interno del corpo vertebrale per creare una cavità (Fig. 6) che successivamente viene riempita col cemento (Fig. 7). In questo modo con la cifoplastica si restituisce, per quanto possibile da caso a caso, "altezza" al corpo vertebrale in quella che può essere definita una sorta di "riduzione" della frattura (Fig. 8). Ciò non è ovviamente possibile con la vertebroplastica, neppure utilizzando il cosiddetto sistema Confidence. Quest'ultima pertanto è utilizzata principalmente nei casi in cui non sia necessario "ridurre" la frattura.

In sintesi queste due relativamente recenti procedure mini-invasive si sono dimostrate particolarmente utili nel diminuire la sintomatologia dolorosa dovuta al crollo di uno o più corpi vertebrali (12). Rispetto al trattamento conservativo con immobilizzazione e scarico vertebrale, le metodiche di plastica vertebrale hanno permesso la riduzione, e in molti casi l'eliminazione, dell'uso di analgesici e quindi delle loro complicanze (13). Nel contempo esse hanno migliorato significativamente la qualità di vita dei pazienti sia dal punto di vista fisico che psico-sociale $(14,15)$. Infine la plastica vertebrale consente anche un migliore allineamento vertebrale, riducendo così le complicanze relative alla progressiva cifotizzazione post-fratturativa e in particolare la deformità vertebrale residua che è di per sé significativo fattore di rischio per successive cadute e quindi nuove fratture (16).

Cifo- e vertebroplastica sono quindi tecniche chirurgiche semplici e precise, caratterizzate da invasività minima $\mathrm{e}$ complicanze infrequenti. Una revisione della Food and Drug Administration ha mostrato che è una metodica sicura e le complicanze sono infrequenti se la procedura è eseguita da un operatore esperto (17).

raffaele.partescano@asf.toscana.it

\section{Bibliografia}

1. Alem Am, Sherrard DJ, Gillen DL, et al. Increased risk of hip fracture among patiens with end-stage renal disease. Kidney Int 2000; 58: 396-9.

2. Isaia GC, Tamone $\mathrm{C}$, Ravazzoli M. La patologia fratturativa nei diversi gradi di insufficienza renale cronica. G Ital Nefrol 2008; 25: 57-65.

3. Kado DM, Browner WS, Palermo L, et al. Vertebral fractures and mortality in older women: a prospective study. Study of Osteoporotic Fractures Research Group. Arch Intern Med 1999; 14; 159(11): 1215-20.

4. Silverman SL. The clinical consequences of vertebral compression fracture. Bone 1996;13 (Suppl. 2): S27-31.
5. Lieberman IH, Dudeney S, Reinhardt MK, Bell G. Initial outcome and efficacy of Kyphoplasty in the treatment of painful osteoporotic vertebral compression fractures. Spine 2001; 26: 1631-8.

6. Galibert P, Dearamond H, Rosat P, Le Gars D. Note preliminaire sur le traitement des angiomes vertebraux par vertebroplastie percutanée. Neurochirurgie 1987; 33: 166-8.

7. Garfin SR, Yuan HA, Reiley MA. New technologies in spine. Kyphoplasty and vertebroplasty for the treatment of painfulosteoporotic compression fractures. Spine 2001; 26: 1511-5.

8. Gaitanis IN, Hadjipavlou AG, Katonis PG, Tzermiadianos MN, Pasku DS, Patwardhan AG. Ballon Kyphoplasty for the treatment of pathological vertebral compressive 
fractures. Eur Spine J 2005; 250-60.

9. Garfin SR et al. Ballon Kyphoplasty for sintomatic vertebral body compression fractures results in rapid, significant and sustained improvements in back pain, function and quality of life for elderly patients. Spine 2006; 31(19): 2213-20.

10. Phillips FM. Minimally invasive treatments of osteoporotic vertebral compression fractures. Spine 2005; 28(Suppl): S45-53.

11. Steinmann J, Tingry CT, Cruz G, Day Q. Biomechanical comparison of unipeduncolar versus bipeduncolar kyphoplasty. Spine 2005; 201-205.

12. Partescano R. La cifoplastica nel paziente anziano con crollo vertebrale osteoporotico. Agg. CIO 2008; 14(2): 39-41.

13. Wardlaw D, Boonen S, Bastian L, Van Meirhaeghe J. An international multicenter randomized comparison of ballon kyphoplasty and nonsurgical care in patients with acute vertebral body compression fractures. Eur Spine J 2007; 16 (Suppl 1): S16.

14. Massari L, Caruso G, Paganelli M, Kiokia E, Artioli F. Qualità della vita dopo vertebroplastica e cifoplastica per il trattamento delle fratture vertebrali osteoporotiche da compressione. GIOT 2006; 32: 104-10.

15. McKiernan F, Faciszewski T., Jensen R. Quality of life following vertebroplasty. J Bone Join Surgery 2004; 86: 2600-9.

16. Villarraga ML, Bellezza AJ, Harrigan TP, Cripton PA, Kurtz SM, Edidin AA The biomechanical effects of kyphoplasty on treated and adjacent nontreated vertebral bodies. J Spin Dis Tec 2005;18: 84-91.

17. Nussbaum DA, Gailloud P, Murphy K. A review of complication associated with vertebroplasty and kyphoplasty as reported to the Food and Drug Administration Medical Device related web site. J Vasc Interv Radiol 2004; 15: 1185-92. 